\title{
A CHARACTERIZATION OF PRINCIPAL CONGRUENCES OF DE MORGAN ALGEBRAS AND ITS APPLICATIONS
}

\author{
H.P. Sankappanavar
}

\begin{abstract}
In this paper a characterization of principal congruences of De Morgan algebras is given and from it we derive that the variety of De Morgan algebras has DPC and CEP. The characterization is then applied to give a new proof of Kalman's characterization of subdirectly irreducibles in this variety and thus to obtain the representation theorem for De Morgan algebras first proved by Kalman and independently, using topological methods, by Bialynicki-Birula and Rasiowa. From this representation it is deduced that finite De Morgan algebras are not the only ones with Boolean congruence lattices. Finally it is shown that the compact elements in the congruence lattice of a De Morgan algebra form a Boolean sublattice.
\end{abstract}

\section{§1. INTRODUCTION,}

Principal congruences have turned out to be a useful tool, especially after the publication of Day 1971, in obtaining some interesting in formation about a given variety (equational class) of algebras. For example, the "equational" characterization of principal congruences of distributive lattices - first given by Grätzer and Schmidt (see Grätzer 1971, Theorem 3, p. 87) - has been used, among other things, to characterize subdirect irreducibility (Example 3 in Grätzer 1971); and it also implies that the variety of distributive lattices has definable principal congruences(DPC) (see $\$ 4$ below for definition) and has congruence extension property (CEP). (This is immediate via Day 1971.) Lasker 1973 found a similar characterization in the case of distributive pseudo-complemented lattices and used it to deduce CEP and DPC. Recently we observed in Sankappanavar 197+ b that his characterization could also be used to give a very simple proof of the characterization by Lakser (see Grätzer 1971, Theorem 7, p. 178) of subdirectly irreducible distributive pseudocomplemented lattices. To cite still another example, principle congruences of pseudo-complemented semilattices are characterized in Sankappanavar 197+ a and DPC and CEP are deduced; moreover, this characterization is applied in Sankappanavar $197+b$ to give a new direct proof of a result, due to Jones, characterizing subdirectly irreducible ones.

In this paper a characterization of principal congruences of De Morgan aigebras is given. From this it follows that the variety of De Morgan algebras has DPC and CEP. This characterization is then applied to give a new proof of $\mathrm{Kal}$ man's characterization of subdirectly irreducibles in this variety and thus to obtain the representation theorem for De Morgan algebras first proved by Kalman and later, using topological methods, by Bialynicki-Birula and Rasiowa. From this it 
is deduced that finite De Morgan algebras are the only ones with Boolean congruence lattices. Finally it is shown that the compact elements in the congruence lattice of a De Morgan algebra form a Boolean sublattice.

Recall that a De Morgan aigebra is an algebra $\langle A ; \wedge, V, \sim, 0,1\rangle$ of type $\langle 2,2,1,0,0\rangle$ such that $\langle A ; \wedge, \vee, 0,1\rangle$ is a bounded distributive lattice satisfying the identities $\sim \sim x=x$ and $\sim(x \vee y)=\sim x \wedge \sim y$. (We refer to $\sim$ as the De Morgan negation. $)^{\dagger}$ For a systematic study of these algebras see Balbes and Dwinger 1974 and Rasiowa 1974. Throughout this paper $A$ denotes an arbitrary De Morgan algebra. For the notation we refer the reader to Balbes and Dwinger 1974 and Grätzer 1971. We let $\theta(a, b)$ and $\theta_{\text {Lat }}(a, b)$ denote respectively the principal De Morgan algebra congruence and the principal lattice congruence on $A$ generated by the pair $(a, b)$ in $A \times A$. $B(A)$ denotes the set of complemented elements of $A$, while $C(A)$ consists of those elements $a$ of $A$ such that the Booiean complement $a^{\prime}$ in $A$ coincides with the De Morgan negation $\sim a$. It is clear that $C(A) \subseteq B(A)$; and the equality holds iff $A$ is a Boolean algebra. Finally we let $\operatorname{con} A$ to denote the congruence lattice of $A$ and recall that it is a distributive algebraic lattice.

\section{§ 2. PRINCIPAL CONGRUENCES,}

In this section we shall give a characterization of principal congruences of a De Morgan algebra. The following Lemma - whose proof is straightforward - is crucial for the rest of the paper.

LEMMA 2.1. Let $a, b \in A$ with $a \leqslant b$. Then

$$
\theta(a, b)=\theta_{\text {Lat }}(a, b) \vee \theta_{\text {Lat }}(\sim b, \sim a) \text {. }
$$

THEOREM 2.2. Let $a, b \in A$ such that $a \leqslant b$. For $x, y$ in $A,(x, y) \in$ $\theta(a, b)$ iff the following identities are true in $A:$

1) $x \wedge a \wedge \sim b=y \wedge a \wedge \sim b$

2) $(x \wedge a) \vee \sim a=(y \wedge a) \vee \sim a$

3) $(x \vee b) \wedge \sim b=(y \vee b) \wedge \sim b$

4) $x \vee b \vee \sim a=y \vee b \vee \sim a$,

or equivalently the identities 1), 2'), 3') and 4) are true in A, where

2') $(x \vee \sim a) \wedge a=(y \vee \sim a) \wedge a$, and

$\left.3^{1}\right)(x \wedge \sim b) \vee b=(y \wedge \sim b) \vee b$.

PROOF. Let us denote by $[a]$ the element in the distributive lattice $A / \theta$ Lat $\left(\sim b^{*}, \sim a\right)$ corresponding to an element $a \in A$. Then by Lemma 2.1 we have

+ These algebras were first studied in Kalman 1958 (actually in his thesis 1955) (but without 0 and 1) as a common abstraction of Boolean algebras and latticeordered groups and by Bialynicki-Birula and Rasiowa under the name of quasiBoolean algebras. These algebras are related to constructive logic with strong negation. 
for $x, y \in A$,

$$
\begin{aligned}
(x, y) \in \theta(a, b) \quad & \text { iff }([x],[y]) \in \theta_{\text {Lat }}([a],[b]) \text { in } A / \theta_{\text {Lat }}(\sim b, \sim a) \\
& \text { iff }[x] \wedge[a]=[y] \wedge[a] \text { and }[x] \vee[b]=[y] \vee[b] \\
& \text { iff }(x \wedge a, y \wedge a) \in \theta_{\text {Lat }}(\sim b, \sim a) \text { and }(x \vee b, y \vee b) \in \theta_{\text {Lat }}(\sim b, \sim a) \\
& \text { iff the identities } 1)-4) \text { are true in } A .
\end{aligned}
$$

Similarly by considering $A / \theta_{\text {Lat }}(a, b)$ it is readily seen that $(x, y) \in \theta(a, b)$ iff 1), $\left.\left.2^{1}\right), 3^{\prime}\right)$ and 4) hold in $A$ and thus the proof is complete. lent:

COROLLARY 2.3. Let $x, y, a \in A$. Then the following conditions are equiva-

a) $(x, y) \in \theta(a, 1)$

b) $(x \wedge a) \vee \sim a=(y \wedge a) \vee \sim a$

c) $(x \vee \sim a) \wedge a=(y \vee \sim a) \wedge a$.

In particular for $a \in C(A)$ we have $(x, y) \in \theta(a, 1)$ iff $x \wedge a=y \wedge a$ iff $x \vee \sim a=y \vee \sim a$.

(We just remark here that this corollary includes the well-known characterization of principal congruences of Boolean algebras.)

COROLLARY 2.4. Let $a \in A$. Then $\theta(a, a \vee \sim a)=\theta_{\text {Lat }}(a, a \vee \sim a)$. In other words, $\quad x, y\} \in \theta(a, a \vee \sim a)$ iff $x \wedge a=y \wedge a$ and $x \vee a \vee \sim a=y \vee a \vee \sim a$. In particular, if $a \leqslant \sim a$ then $(x, y) \in \theta(a, \sim a)$ iff $x \wedge a=y \wedge a$ and $x \vee \sim a=y \vee \sim a$.

PROOF. Observe $(\sim(a \vee \sim a), \sim a) \in \theta_{\text {Lat }}(a, a \vee \sim a)$

COROLLARY 2.5. Let $a \in A$. Then the following are equivalent. ence on $A$,

i) The lattice congruence $\theta_{\text {Lat }}(a, 1)$ on $A$ is a De Morgan algebra congru-

ii) $\sim a$ is the Boolean complement of $a$ in $A$ li.e. $\sim_{a} \in C(A)$ ).

PROOF. From $i)$ and $(\sim a, 0) \in \theta(a, 1)$ we see that $(\sim a, 0) \in \theta_{\text {Lat }}(a, 1)$ which implies $\sim a \wedge a=0$, thus yielding $i j)$. On the other hand, from $i j$ ) and Corollary 2.3 we have $(\sim a, 0) \in \theta_{\text {Lat }}(a, 1)$, thius $\left.i\right)$ is immediate.

Thus the property i) in the above corollary is characteristic of Boolean algebras among De Morgan algebras. It also follows from the preceding corollary that the condition (2) of Lemma 4.3 in Cignoli 1975 can be improved to: For every $a \in B(A)$ the lattice congruence $\theta_{\text {Lat }}(a, 1)$ is also a De Morgan algebra congruence on $A$.

\section{§3. APPLICATIONS,}


Following Baldwin and Berman 1975 we say that a variety $v$ has definable principal congruences (DPC) iff there is a formula $\phi(u, v, r, s)$ in the first order language of $V$ so that for all $A \in V$ and $a, b, c, d \in A, \phi(c, d, a, b)$ holds in $A$ iff $(c, d) \in \theta(a, b)$. Regarding the usefulness of $D P C$ and varieties with $D P C$ the reader may refer to, for example, Baldwin and Berman 1975, Mckenzie 1978 . Grätzer 1971, Lakser 1973 and Sankappanavar 197+ b.

Thus the following Theorem is immediate from Theorem 2.2.

THEOREM 3.1. The variety of De Morgan algebras has DPC.

Day 1972 proved that if an equational class has the property that in each of its members the condition $(x, y) \in \theta(a, b)$ is determined by a set of equations involving only $x, y, a, b$ then the congruence extension property (see Grätzer 1971 for definition) holds in that class. In the case of De Morgan algebras the reader can easily write down such equations using our Theorem 2.2 for $x, y, a, b$ in $A$ (where $a, b$ are not necessarily comparable). Thus we have

THEOREM 3.2. The variety of De Morgan algebras has the congruence extension property.

It may be remarked here that Theorems 3.1 and 3.2 could be obtained by more involved methods. For example Theorem 3.2 is a consequence of results in cignoli 1975 and Banaschewski 1969 and then an existential proof of Theorem 3.1 is obtained from Theorem 3.2 and the (known) fact that every finitely generated De Morgan algebra is finite, together with a result in Baldwin and Berman 1975, while our proof gives an explicit defining formula.

\section{SUBDIRECTLY IRREDUCIBLE DE MORGAN ALGEBRAS,}

Kalman 1958 characterized the subdirectly irreducible De Morgan algebras and thereby obtained the complete description of the lattice of subvarieties of the variety of De Morgan algebras and a representation theorem. Later, using topological methods Bialynicki-Birula and Rasiowa 1958 (see also Balbes and Dwinger 1974 obtained the same representation theorem.

We shall now apply Theorem 2.2 to give new proofs of these results. For this we need the following observation.

LEMMA 3.3. Let $A$ be a De Morgan algebra of height $\geqslant 3$ and with $|C(A)|=2$. Then there is an element $a \neq 0$ in $A$ such that $a<\sim a$.

PROOF. We may suppose that there are elements $x_{1}, x_{2}$ in $A$ such that $0<$ $x_{1}<x_{2}<1$. We may further assume that each of $\sim x_{1}$ and $\sim_{2}$ is incomparable with each of $x_{1}$ and $x_{2}$ since in all other cases the Lema is trivially verified. Now set $a=\left(x_{1} \vee \sim x_{2}\right) \wedge x_{2} \wedge \sim x_{1}$ and we claim that a has all the desired properties. Observe that $\sim a=x_{1} \vee \sim x_{2} \vee\left(\sim x_{1} \wedge x_{2}\right)$ and hence $a \leqslant v a$; furthermore if $a=\sim a$ then $x_{1} \vee \sim x_{2}=x_{2} \wedge \sim x_{1}$ and hence $\sim x_{2} \leqslant x_{2}$ which contradicts our supposition and thus $a<\sim a$. Finally if $a=0$ then noting that $a=\left(x_{1} \wedge \sim x_{1}\right) \vee\left(\sim x_{2} \wedge x_{2}\right)$ we see that $x_{1} \wedge \sim x_{1}=0$ and hence $x_{1} \in$ $C(A)$ which is a contradiction to the hypothesis. Thus our claim is proved and 
hence the Temma.

The following three De Morgan algebras 2, 3 and 4 given in figure 1 , where $\sim a=a, \sim b=b$ and $\sim c=c$, play a very special role in the theory of De Morgan algebras. We now apply our results to give a simple proof of Kalman's result mentioned earlier. Observe that $B(4)=\{0, b, c, 1\}$ while $C(4)=\{0,1\}$.

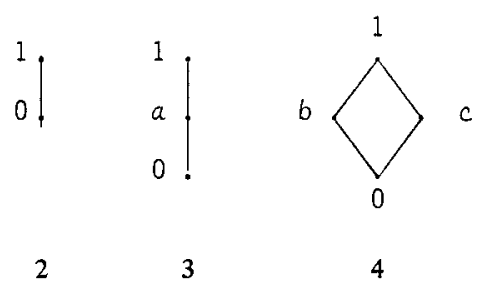

(Figure 1)

THEOREM 3.4. (Kalman 1958) Let A be non-trivial. Then the following are equivalent:
1) $A$ is simple.
2) $A$ is subdirectly irreducible.
3) $A$ is of height $<3$ and $C(A)=\{0,1\}$
4) $A$ is either 2 or 3 or 4 .

PROOF, 2) $\Rightarrow 3$ ) is the only non-trivial implication, and we shall prove it here. Suppose $A$ is subdirectly irreducible. If $C(A)$ has an element different from 0 and 1 then from Corollary 2.5 it follows that $\theta(b, 1) \wedge \theta(\sim b, 1)=\Delta$ (the least element in Con $A$ ), which is a contradiction and hence $C(A)=\{0,1\}$. Next suppose the height of $A \geqslant 3$. Then by the preceding Lemma there is an element $a$ in $A$ such that $a<\sim a$ and $a \neq 0$. But then from Corollaries 2.3 and 2.4 it follows that $\theta(a, \sim a) \wedge \theta(\sim a, 1)=\Delta$ which is again a contradiction and so the height of $A$ is less than 3 . Thus (3) is true and the theorem is proved.

In view of the famous Birkhoff's theorem the following corollaries are immediate. Let $V(A)$ denote the variety generated by $A$.

COROLLARY 3.5. Everu non-trivial De Morgan algebra $A$ is a subdirect product of simples lviz. 2,3 and 4 ).

COROLLARY 3.6. (Kalman 1958; Bialynicki-Birula and Rasiowa 1957) A is a subalgebra of a direct power of 4 .

COROLLARY 3.7. (Kalman 1958) The lattice of subvarieties of De Morgan algebras is a $f$-eiement chain consisting of V(1) (trivial cigebras), V'2) (Boolean algebras), V(3) (so-called Kleene algebras) and V(4) (all De Morgan algebras).

Finally combining Corollary 3.4 with Day 1972, Theorem 4.1, one readily obtains the following Corollary which was first obtained in Cignoli 1975 by giving 
an explicit characterization of injectives.

COROLLARY 3.8. (Cignoli 1975) $V(4)$ and $V(3)$ have enoug. injectives and hence have the amalgamation property.

\section{CONGRUENCE LATTICES,}

We shall now apply our previous results to obtain some information about the structure of congruence lattices. We have the following theorem characterizing the De Morgan aigebras with Boolean congruence lattices.

\section{THEOREM 3.8. COnA is Bookean iff $A$ is finite.}

PROOF, Since A is semisimpie, from a result of Tanaka 1952 (or see Burris 1975 , Corollary 2.6) it follows that Con $A$ is a Boolean lattice iff any two elements of $A$ are equal on all but finitely many factors, and the latter is true iff $A$ is finite.

In the rest of the paper our concern is to show that the compact elements in Con A form a Boolean sublattice of Con A. It is well-known that in a distributive lattice the meet of two principal congruences is a principal congruence (see Lakser 1973 for example). In fact, if $a \leqslant b$ and $c \leqslant d$ then $\theta_{\text {Lat }}(a, b) \wedge$ $\theta_{\text {Lat }}(c, d)=\theta_{\text {Lat }}(b \wedge d \wedge\langle a \vee c|, b \wedge d)$. However, in a De Morgan algebra this property may not hoid in general, but we still have the following useful information.

LEMMA 3.9. Let $a, b, c, d \in A$ with $a \leqslant b$ and $c \leqslant d$. Then

$$
\theta(a, b) \wedge \theta(c, d)=\theta(a \vee c, a \vee c \vee(b \wedge d)) \vee \theta(a \vee \sim d, a \vee \sim d \vee(b \wedge \sim c))
$$

PROOF, Using Lemma 2.1 and distributivity of $\operatorname{Con} A$ we have

$$
\begin{aligned}
\theta(a, b) \wedge \theta(c, d)= & {\left[\theta_{\text {Lat }}(a, b) \vee \theta_{\text {Lat }}(\sim b, \sim a)\right] \wedge\left[\theta_{\text {Lat }}(c, d) \vee \theta_{\text {Lat }}(\sim d, \sim c)\right] } \\
= & {\left[\theta_{\text {Lat }}(a, b) \wedge \theta_{\text {Lat }}(c, d)\right] \vee\left[\theta_{\text {Lat }}(a, b) \wedge \theta_{\text {Lat }}(\sim d, \sim c)\right] } \\
& \vee\left[\theta_{\text {Lat }}(\sim b, \sim a) \wedge \theta_{\text {Lat }}(c, d)\right] \vee\left[\theta_{\text {Lat }}(\sim b, \sim a) \wedge \theta_{\text {Lat }}(\sim d, \sim c)\right] \\
= & \theta_{\text {Lat }}(b \wedge d \wedge(a \vee c), b \wedge d) \vee \theta_{\text {Lat }}(b \wedge \sim c \wedge(a \vee \sim d), b \wedge \sim c) \\
& \vee \theta_{\text {Lat }}(\sim a \wedge d \wedge(\sim b \vee c), \sim a \wedge d) \vee \theta_{\text {Lat }}(\sim a \wedge \sim c \wedge(\sim b \vee \sim d), \sim a \wedge \sim c) .
\end{aligned}
$$

Recall that $\theta_{\text {Lat }}(x \wedge y, x)=\theta_{\text {Lat }}(y, x \vee y)$, and thus we have

$$
\begin{aligned}
\theta(a, b) \wedge \theta(c, d)= & \theta_{\text {Lat }}(a \vee c,(b \wedge d) \vee a \vee c) \vee \theta_{\text {Lat }}(a \vee \sim d, a \vee \sim d \vee(b \wedge \sim c)) \\
& \vee \theta_{\text {Lat }}(\sim a \wedge d \wedge(\sim b \vee c), \sim a \wedge d) \vee \theta_{\text {Lat }}(\sim a \wedge \sim c \wedge(\sim b \vee \sim d), \sim a \wedge \sim c)
\end{aligned}
$$




$$
=\theta(a \vee c, a \vee c \vee(b \wedge d)) \vee \theta(a \vee \sim d, a \vee \sim d \vee(b \wedge \sim c))
$$

Thus the Lemma is proved.

LEMMA 3.10. Every principal congruence on A has a complement which is compact in Con $A$. In fact if $a, b \in A$ and $a \leqslant b$ then

$$
\theta(a, b)^{\prime}=\theta(\sim a \vee b, 1) \vee \theta(b \wedge \sim b, \sim b) \vee \theta(\sim a, \sim a \vee a)
$$

PROOF, Observe that

$$
\begin{aligned}
& (0, \sim b \wedge a) \in \theta(\sim a \vee b, 1),(\sim b \wedge a, \sim b \wedge b) \in \theta(a, b), \\
& (\sim b \wedge b, \sim b) \in \theta(b \wedge \sim b, \sim b),(\sim b, \sim a) \in \theta(a, b), \\
& (\sim a, \sim a \vee a) \in \theta(\sim a, \sim a \vee a),(\sim a \vee a, \sim a \vee b) \in \theta(a, b) \text { and } \\
& (\sim a \vee b, 1) \in \theta(\sim a \vee b, 1) .
\end{aligned}
$$

Thus if $\Phi$ denotes the congruence on the right side of the above equation then we just observed that $\theta(a, b) \vee \Phi=\theta(0,1)$. Finally using the previous Lemma it is straightforward to verify that $\theta(a, b) \wedge \Phi=\Delta$.

The following theorem reveals the nice structure of congruence lattices of De Morgan algebras.

THEOREM 3.11. Con A is a distributive algebraic lattice whose compact elements form a Boolean sublattice.

PROOF, From Lemma 3.9 and distributivity of Con $A$ it readily follows that the meet of compact elements is compact in Con $A$ and thus the compact elements in Con $A$ form a sublattice. Finally the fact that this sublattice is Boolean follows from Lemma 3.10 , thus the proof is complete.

Finally we remark that in view of Theorem 3.8 the converse to the above theorem is true if $A$ is finite, however it is not known if it holds in general.

\section{ACKNOWLEDGEMENT,}

The author would like to thank the organizing committee of the Fourth LatinAmerican Symposium on Mathematical Logic for inviting him to participate in the Symposium. The final version of this paper was written during January 1979 when the author was visiting the Department of Pure Mathematics, University of Water 100, Waterioo, Ontario, Canada, to whom the author is grateful. Finaliy he is speclaily thankful to Professor Stanley Burris who kindly invited the author to present the results of this paper in his Logic Seminar and who has always been the source of inspiration to the author. 


\section{REFERENCES,}

R. Balbes and $P$. Dwinger

1974 Distributive Lattices, University of Missouri Press, Columbia.

J. Baldwin and J.T. Berman

1975 The number of subdirectly irreducible algebras in a variety. Alg. Univ. vol. 5, pp. $379-389$.

B. Banaschewski

1969 Injectivity and essential extensions in equational classes of algebras, Proceedings of the Conference in Universal Algebra, Queen's University, Kingston.

A. Bialynicki - Birula and H. Rasiowa

1957 On the representation of quasi-Boolean algebras, Bul1. Acad. Pol. Sci.CI. III 5, pp. $259-261$.

1958 On constructible falsity in the constructive logic with strong negation, Colloq. Math. vol. 6, pp. $287-310$.

S. Burris

1975 Separating sets in modular lattices with applications to congruence lattices, Alg. Univ. vol. 5, pp. 213-223.

R. Cignoli

1975 Injective De Morgan and Kleene algebras, Proc. Amer. Math. Soc, vol. 47 , pp. $269-278$.

1978 The lattice of glabal sections of sheaves of chains over Boolean spaces, Alg. Univ. vol. 8, pp. $357-373$.

A. Day

1971 A note on the congruence extension property, Alg. Univ. vol. 1, pp. 234 235.

1972 Injectivity in equational classes of algebras, Can. J. Math. vol. 24, pp. $209-220$.

G. Grätzer

1971 Lattice Theory: First concepts and distributive lattices, Freeman, San Francisco.

J. Kalman

1958 Lattices with involution, Trans. Amer. Math. Soc. vol. 87, pp. 485-491. 


\section{H. Lakser}

1973 Principal congruences of pseudocamplemented semilattices, Proc. Amer. Math. Soc., vol. $37, \mathrm{pp} .32-36$.

\section{R. McKenzie}

1978 Para primal varieties: A study of finite axiomatizability and definable principal congruences in locally finite varieties, Alg. Univ. vol. 8, pp. $336-348$.

H. Rasiowa

1974 An algebraic approach to non-classical logics, North-Holland, Ams terdam.

H.P. Sankappanavar

$197+$ a Principal congruences of pseudocomplemented semilattices and congruence extension property, Proc. Amer. Math. Soc. (to appear).

$197+b$ Remarks on subdirectly irreducible pseudocomplemented semilattices and distributive pseudocomplemented lattices. (Preprint).

\section{T. Tanaka}

1952 Canonical subdirect factorization of lattices, J. Sci. Hiroshima Univ.Ser. A-1, vol. 16, pp. $239-246$.

Instituto de Matematica

Universidade Federal da Bahia

Salvador, Bahia, Brazil.
Department of Pure Mathematics

University of Waterloo

Waterioo, Ontario, Canada. 